\title{
Clique-width and the speed of hereditary properties
}

\author{
Peter Allen* $\quad$ Vadim Lozin ${ }^{\dagger} \quad$ Michaël Rao ${ }^{\ddagger}$ \\ Submitted: Sep 30, 2008; Accepted: Mar 5, 2009; Published: Mar 13, 2009 \\ Mathematics Subject Classification: 05A16, 05C75, 05C78
}

\begin{abstract}
In this paper, we study the relationship between the number of $n$-vertex graphs in a hereditary class $\mathcal{X}$, also known as the speed of the class $\mathcal{X}$, and boundedness of the clique-width in this class. We show that if the speed of $\mathcal{X}$ is faster than $n ! c^{n}$ for any $c$, then the clique-width of graphs in $\mathcal{X}$ is unbounded, while if the speed does not exceed the Bell number $B_{n}$, then the clique-width is bounded by a constant. The situation in the range between these two extremes is more complicated. This area contains both classes of bounded and unbounded clique-width. Moreover, we show that classes of graphs of unbounded clique-width may have slower speed than classes where the clique-width is bounded.
\end{abstract}

Keywords: Clique-width; Hereditary class of graphs; Speed of hereditary classes

\section{Introduction}

Clique-width is a graph parameter which is of primary importance in algorithmic graph theory because many problems being NP-hard in general admit polynomial-time solutions when restricted to a class $\mathcal{X}$ of graphs where the clique-width is bounded by a constant [9]. In the study of clique-width we may assume, without loss of generality, that $\mathcal{X}$ is a hereditary class of graphs, i.e., a class closed under taking induced subgraphs, because the clique-width of a graph cannot be less than the clique-width of any of its induced subgraphs.

In a recent line of research, it was shown that the growth of the number $X_{n}$ of $n$-vertex graphs in a hereditary class $\mathcal{X}$, also known as the speed of the class, is far from arbitrary. Specifically, the rates of growth constitute discrete layers. Alekseev [1] and Scheinerman

*DIMAP and Mathematics Institute, University of Warwick, Coventry CV4 7AL, UK. P.D.Allen@warwick.ac.uk. This author gratefully acknowledges the support of DIMAP - Centre for Discrete Mathematics and its Applications at the University of Warwick

†DIMAP and Mathematics Institute, University of Warwick, Coventry CV4 7AL, UK. V.Lozin@warwick.ac.uk. This author gratefully acknowledges the support of DIMAP

${ }_{\ddagger}^{\ddagger}$ LIRMM, Montpellier, France. Michael.Rao@lirmm.fr 
and Zito [20] discovered five lower layers: constant, polynomial, exponential, factorial and superfactorial. These correspond to classes $\mathcal{X}$ whose speeds are respectively constant, polynomial, bounded above and below by exponential functions of $n$, bounded above and below by functions of the form $n^{c n}$, and of the form $\Omega\left(n^{c n}\right)$ for every $c$.

The structure of graphs in the first three layers is rather simple, implying boundedness of the clique-width in any class from these layers. However the structure of a class $\mathcal{X}$ whose speed is factorial is not so simple. Balogh, Bollobás and Weinreich $[3,5]$ gave a precise classification of all the possible speeds, together with the corresponding graph structures, up to the Bell number $B_{n}$; but they were unable to give any such results either for the remainder of the factorial layer or for the superfactorial graph classes with speeds of the

form $2^{\left.o\left(\begin{array}{l}n \\ 2\end{array}\right)\right)}$. Indeed, in [4] they were able to show that some properties with speeds in these ranges have exceptionally badly behaved speeds.

In the present paper we show that the clique-width is bounded in any hereditary class whose speed does not exceed the Bell number $B_{n}$. On the other hand, we prove that for much of the remaining factorial layer, and in any superfactorial class of graphs, the clique-width is unbounded. This perhaps provides some reason why Balogh, Bollobás and Weinreich were unable to extend their characterisation: the structure of graphs in these higher speed classes is much more complex.

However there is no 'boundary speed' separating classes with bounded and unbounded clique-width; we exhibit properties of unbounded clique-width whose speeds are strictly slower than those of various classes of bounded clique-width.

Let $\mathcal{T}(k)$ be the hereditary class of graphs with clique-width at most $k$. We note that $\mathcal{T}(1)$ is the class of disjoint unions of cliques and so has speed equal to the Bell number; for larger $k$ we will show that

$$
n !\left(\frac{2^{\frac{k-5}{2}}}{k-2}\right)^{n} \leq T(k)_{n} \leq n !\left(\left(2 k^{2}\right)^{2 k^{2}}\right)^{n} .
$$

The organization of the paper is as follows. In Section 2 we give some preliminary information related to the topic of the paper. In Section 3, we show that the cliquewidth is unbounded in any superfactorial class of graphs, and in Section 4, we prove that the clique-width is bounded in any hereditary class whose speed does not exceed the Bell number $B_{n}$. Section 5 is devoted to graph classes between the two boundaries. In particular, in Section 5.1 we describe a class of graphs of bounded clique-width whose speed is faster than the speed of two classes of unbounded clique-width described in Section 5.2.

\section{Preliminaries}

Unless specified, all graphs in this paper are undirected, without loops and multiple edges. For a graph $G$, we denote by $V(G)$ and $E(G)$ the vertex set and the edges set of $G$ respectively. The complement of $G$ is denoted $\bar{G}$. The neighborhood of $v \in V(G)$ is the set of vertices adjacent to $v$. For a subset $U \subseteq V(G)$, we denote by $G[U]$ the subgraph 
of $G$ induced by $U$, i.e., the graph with vertex set $U$ and two vertices being adjacent in $G[U]$ if and only if they are adjacent in $G$. Given a set of graphs $M$, we say that $G$ is $M$-free if $G$ does not contains induced subgraphs isomorphic to graphs in $M$. As usual, we denote by $C_{k}$ a chordless cycle with $k$ vertices and by $K_{n, m}$ a complete bipartite graph with parts of size $n$ and $m$.

The notion of clique-width of a graph was introduced by Courcelle, Engelfriet and Rozenberg in [8] and is defined as the minimum number of colours needed to construct the graph by means of the following graph operations:

- Create a vertex $v$ with colour $i: i(v)$.

- Take the disjoint union of two previously constructed graphs $G$ and $H$ (preserving the vertex colours): $G \oplus H$.

- Put an edge from each vertex of colour $i$ to each vertex of colour $j: \eta_{i, j}$.

- Recolour all vertices of colour $i$ to colour $j: \rho_{i \rightarrow j}$.

Every graph can be defined by an algebraic expression using the four operations above. For instance, the graph consisting of two adjacent vertices $x$ and $y$ can be defined by the expression $\eta_{1,2}(1(x) \oplus 2(y))$, and the cycle $C_{5}$ on vertices $a, b, c, d, e$ (listed along the cycle) can be defined by the following expression:

$$
\eta_{4,1}\left(\eta_{4,3}\left(4(e) \oplus \rho_{4 \rightarrow 3}\left(\rho_{3 \rightarrow 2}\left(\eta_{4,3}\left(4(d) \oplus \eta_{3,2}\left(3(c) \oplus \eta_{2,1}(2(b) \oplus 1(a))\right)\right)\right)\right)\right)\right) .
$$

This example suggests the idea of how to construct any cycle with at most 4 different colours. Unfortunately, in general, the clique-width can take arbitrarily large values. In this paper, we study the relationship between boundedness of the clique-width in a certain class of graphs and the number of $n$-vertex graphs in this class. To this end, we recall the following known facts.

In [10], it was shown that the clique-width of a graph cannot be less than the cliquewidth of any of its induced subgraphs. More generally, denoting the clique-width of a graph $G$ by $\mathrm{cw}(G)$, we have

Lemma 1 For any graph $G$,

$$
\mathrm{cw}(G)=\max \{\mathrm{cw}(H) \mid H \text { is a prime induced subgraph of } G\} \text {. }
$$

The notion of prime graph was introduced in the study of modular decomposition. To define this notion, let us say that a vertex $v \in V(G)$ distinguishes a subset $U \subseteq V(G)-\{v\}$ if $v$ has both a neighbor and a non-neighbor in $U$. A module in a graph is a subset of vertices indistinguishable for the vertices outside the subset. A module $M \subseteq V(G)$ is trivial if $|M|=1$ or $M=V(G)$. A graph $G$ is prime if every module of $G$ is trivial.

Now let us mention several graph operations that do not change the clique-width "too much". The following two lemmas can be found in [10] and [15], respectively. 
Lemma 2 For any graph $G, \operatorname{cw}(\bar{G}) \leq 2 \mathrm{cw}(G)$

Lemma 3 If a graph $G$ is obtained from a graph $H$ by deleting $k$ vertices, then

$$
\mathrm{cw}(G) \leq \mathrm{cw}(H) \leq 2^{k}(\mathrm{cw}(G)+1)
$$

Both these lemmas can be generalized by means of the following two operations.

- Subgraph complementation is the operation of complementing the edges on a subset of the vertices of a graph $G$;

- Bipartite subgraph complementation is the operation of complementing the edges between two disjoint subsets of the vertices of a graph $G$.

Without giving any specific bound on the size of the change of the clique-width under these operations, we simply present the following result proved in [13].

Lemma 4 For a class of graphs $X$ and a nonnegative integer $k$, denote by $X^{(k)}$ the class of graphs obtained from graphs in $X$ by applying at most $k$ subgraph complementations or bipartite subgraph complementations. Then the clique-width of graphs in $X^{(k)}$ is bounded by a constant if and only if it is bounded for graphs in $X$.

The fact that the clique-width of a graph cannot be less than the clique-width of any of its induced subgraphs allows us to be restricted to hereditary graph classes. Every hereditary class (and only hereditary) can be characterized in terms of minimal forbidden induced subgraphs, i.e., a class $\mathcal{X}$ is hereditary if and only if there is a set $M$ such that every graph in $\mathcal{X}$ is $M$-free.

Clearly, not every graph class is hereditary. For instance, the class of trees is not hereditary. However, any class $\mathcal{X}$ can be extended to a hereditary class by adding to $\mathcal{X}$ all induced subgraphs of graphs that are in $\mathcal{X}$. In this way, the class of trees is extended to the class of forests, i.e., graphs without cycles.

\section{Fast speed implying unbounded clique-width}

In this section, we show that hereditary classes with superfactorial speed have unbounded clique-width. More specifically, we prove that the speed of any class of graphs with bounded clique-width is at most factorial.

Theorem 1 The number of graphs on $n$ vertices with clique-width at most $k$ is bounded above by $n ! C^{n}$ for some constant $C$ depending on $k$. 
Proof. If a graph on $n$ vertices has clique-width at most $k$, then there is an expression using $k$ colours that constructs it. We simply bound the number of expressions which could possibly give different graphs. We insist on a convenient form for these expressions.

Suppose that we are in the process of constructing a graph, and have just taken a disjoint union of two coloured graphs. We may now apply edge creating or recolouring operations. We may assume that we perform any edge creation operations first, and then do any necessary recolouring; the number of edge creation operations immediately following a disjoint union operation is thus at most $\left(\begin{array}{l}k \\ 2\end{array}\right)+k$. It is also clear that the recolouring operation $\rho_{i \rightarrow j}$ does nothing if there are no vertices of colour $i$, and is redundant if there are no vertices of colour $j$ (although in many constructions it makes notation simpler to perform some redundant recolouring): so each recolouring operation decreases the number of nonempty colour classes by one. Thus at most $k-1$ recolouring operations may be performed between disjoint unions.

Since each disjoint union joins together two graphs of size at least 1, the number of disjoint union operations is $n-1$. Also, it is obvious that the number of vertex creations is $n$.

Suppose the vertices were unlabelled. In that case the vertex creation operations would simply specify a colour $i$, and the expression would contain the symbols $i$ (for each $1 \leq i \leq k), \eta_{i, j}($ for each $1 \leq i, j \leq k), \rho_{i \rightarrow j}$ (for each $1 \leq i, j \leq k$ ) and $\oplus,($, ), for a total of $k+\left(\begin{array}{l}k \\ k\end{array}\right)+k+2\left(\begin{array}{l}k \\ k\end{array}\right)+3<2 k^{2}$ distinct symbols.

There are at most $n+n-1+2(n-1)\left(\left(\begin{array}{l}k \\ k\end{array}\right)+k\right)+2(n-1)(k-1)<2 k^{2} n$ symbols in the entire expression; thus the number of unlabelled graph with clique-width at most $k$ is at most

$$
\left(2 k^{2}\right)^{2 k^{2} n}=C^{n}
$$

where

$$
C=\left(2 k^{2}\right)^{2 k^{2}} .
$$

There are not more than a factor of $n$ ! more labelled graphs of clique-width $k$ than unlabelled graphs of clique-width $k$, hence

$$
\mathcal{T}(k)_{n} \leq n ! C^{n}
$$

Corollary 1 All superfactorial classes have unbounded clique-width.

Although this is a triviality, it gives one-line proofs of the unboundedness of cliquewidth in various important graph classes (in some cases, replacing long direct proofs of the same fact). We give some examples.

The classes of bipartite, co-bipartite and split graphs are superfactorial and therefore have unbounded clique-width (proved directly in [18]).

The class $X^{p}$ of $K_{p, p}$-free bipartite graphs satisfies (see e.g. [6, 11]):

$$
c_{1} n^{2-\frac{2}{p+1}} \log _{2} n<\log _{2} X_{n}^{p}<c_{2} n^{2-\frac{1}{p}} \log _{2} n,
$$

and thus has unbounded clique-width for each $p \geq 2$; in particular, the class $X_{2}$ of $C_{4}$-free bipartite graphs has unbounded clique-width. 
The result for $C_{4}$-free bipartite graphs has been improved in [17] in the following way. For each odd $k$, the authors present an infinite family of $n$-vertex bipartite graphs of girth (the length of a smallest cycle) at least $k+5$ that have at least $2^{t-k-2} n^{1+\frac{1}{k-t+1}}$ edges, where $t=\left\lfloor\frac{k+2}{4}\right\rfloor$. Consequently, for each odd $k \geq 1,\left(C_{4}, \ldots, C_{k+3}\right)$-free bipartite graphs constitute a superfactorial class and hence are not of bounded clique-width.

Finally we note that the important class of chordal bipartite graphs, (i.e., bipartite graphs containing no induced cycles of length more than four) is superfactorial: Spinrad has shown in [21] that the number of chordal bipartite graphs is $\Omega\left(2^{\Omega\left(n \log ^{2} n\right)}\right)$. Thus this class has unbounded clique-width.

\section{Slow speed implying bounded clique-width}

Now we turn to graph classes with slow speed. We use some structural results obtained in $[1,3,5]$. First, we recall the following characterization of graph classes in the exponential layer that has been presented independently in [1] and [3].

Lemma 5 If $\mathcal{H}$ is a hereditary class of graphs with the speed bounded by an exponentially growing function, then there is a constant $c$ such that the vertex set of any graph in $\mathcal{H}$ can be partitioned into at most $c$ parts such that each part is either a clique or an independent set and between any two parts either no edge is present or every possible edge is present.

In the factorial layer the situation is much more complicated. Structural results are available only for classes with relatively slow speed in this layer. In order to describe these results, let us introduce the following notations. Let $K$ be a graph-with-loops on the vertex set $[k]$, and $G_{k}$ be a simple graph on the same vertex set $[k]$. Let $H^{\prime}$ be the disjoint union of infinitely many copies of $G_{k}$, and for $i=1, \ldots, k$, let $V_{i}$ be the subset of $V\left(H^{\prime}\right)$ containing vertex $i$ from each copy of $G_{k}$. Now we construct from $H^{\prime}$ an infinite graph $H$ on the same vertex set by connecting two vertices $u \in V_{i}$ and $v \in V_{j}$ if and only if $u v \in E\left(H^{\prime}\right)$ and $i j \notin E(K)$ or $u v \notin E\left(H^{\prime}\right)$ and $i j \in E(K)$.

The intent is that $H$ contains infinitely many copies of $G_{k}$ with edges between these copies dictated by $K$. For example, suppose $K$ were the two-vertex graph with two loops, and $G_{2}=K_{2}$. Then $H^{\prime}$ is an infinite matching, and $H$ consists of two infinite cliques between which there is an infinite matching.

Finally, let $\mathcal{P}\left(K, G_{k}\right)$ be the hereditary class consisting of all the finite induced subgraphs of $H$. The following result was proved in [3].

Theorem 2 For any hereditary property $X$ with $X_{n}<n^{(1+o(1)) n}$, there exists

(1) an integer $k$ such that $X_{n}=n^{(1-1 / k+o(1)) n}$ and

(2) a constant $c$ such that for all $G \in X$ there is a set $W \subseteq V(G)$ of at most c vertices so that $G-W$ belongs to a property $\mathcal{P}\left(K, G_{k}\right)$ for some $K$ and $G_{k}$, where $k$ is the constant defined in (1). 
In [5], it was shown that in the range $X_{n} \geq n^{(1+o(1)) n}$ the minimal speed coincides with the Bell number $B_{n}$ and there are exactly two minimal properties of this speed, namely, disjoint union of cliques and their complements.

We now prove the following corollary.

Corollary 2 If the speed of a hereditary graph class $\mathcal{X}$ is at most $B_{n}$ for infinitely many values of $n$, then there is a constant $c=c(\mathcal{X})$ such that the clique-width of any graph in $\mathcal{X}$ is at most $c$.

Proof. If the speed of $\mathcal{X}$ is slower than factorial, then according to Lemma 5 there is a constant $c$ such that any prime graph in $X$ has at most $c$ vertices. Together with Lemma 1 , this proves that the clique-width of graphs in $\mathcal{X}$ is at most $c$.

If $X_{n}<n^{(1+o(1)) n}$, then according to Theorem 2 there exist constants $c$ and $k$ such that when we delete no more than $c$ vertices from any graph $G \in \mathcal{X}$ we obtain a graph $G^{\prime} \in \mathcal{P}\left(K, G_{k}\right)$ for some $K$ and $G_{k}$. From the definition of $\mathcal{P}\left(K, G_{k}\right)$ it follows that $G^{\prime}$ can be obtained by applying at most $k$ times subgraph complementations and bipartite subgraph complementations to a graph $G^{\prime \prime}$ which is the disjoint union of graphs each of which has at most $k$ vertices. Clearly, the clique-width of $G^{\prime \prime}$ is at most $k$. Therefore, by Lemma 4, the clique-width is bounded for $G^{\prime}$ and hence, by Lemma 3, for $G$.

Finally, if the speed of $\mathcal{X}$ is equal to $B_{n}$ for infinitely many values of $n$, then there is $n_{0}$ such that it is equal to $B_{n}$ for all $n \geq n_{0}$, and either for $n \geq n_{0}$ every $n$-vertex graph is a disjoint union of cliques, or for $n \geq n_{0}$ every $n$-vertex graph is the complement of a disjoint union of cliques. In either case there are no graphs in $\mathcal{X}$ with clique-width exceeding $\max \left(n_{0}, 2\right)$.

\section{Between the boundaries}

In this section, we analyze hereditary properties with factorial speed exceeding the Bell number. This area contains many important properties such as line graphs, forests, permutation graphs, interval graphs, planar graphs and even more generally all minor-closed graph classes (other than the class of all graphs) [19]. In some of the classes in this area the clique width is bounded, in some others it is unbounded. The paper [16] contains complete classification of classes of bipartite graphs defined by a single forbidden induced subgraph with respect to bounded/unbounded clique-width, and the paper [2] analyzes the speed of these classes. Comparison of these two papers reveals that for any class of bounded clique-width in this family the speed is at most $n^{n+o(n)}$, while for classes of unbounded clique-width the speed is at least $n^{\frac{3}{2} n+o(n)}$. This observation raises the following interesting question: is there any "boundary speed" with respect to clique-width, i.e., a speed separating classes of bounded clique-width from those where the clique-width is unbounded. In this section we answer this question negatively. First, in Section 5.1 we show that the number of graphs on $n$ vertices with clique-width $k+3$ is at least $n ! c^{n}$ where $c=\frac{2^{\frac{k-2}{2}}}{k+1}$. In particular, the class of graphs of clique-width at most 25 has speed at 
least $n ! 44^{n}$. Then in Section 5.2 we exhibit two classes of unbounded clique-width with speed at most $n ! 28^{n}$.

\subsection{Fast properties of bounded clique-width}

Theorem 3 The number of graphs on $n$ vertices with clique-width $k+3$ is at least $n ! c^{n}$ where $c=\frac{2^{\frac{k-2}{2}}}{k+1}$.

Proof. First observe that using colours $4,5, \ldots, k+3$ we may construct any graph we choose on $k$ vertices, with each vertex given its own unique colour. We may add a special vertex given colour 1 and put this adjacent to all the other vertices. Now we partition the set $\{1,2, \ldots, n\}$ into an ordered sequence of $\left\lfloor\frac{n}{k+1}\right\rfloor$ sets of size $k+1$ and if necessary one smaller set. Let $G_{1}, \ldots, G_{\left\lfloor\frac{n}{k+1}\right\rfloor}$ be coloured graphs on the sets of $k+1$ vertices.

Now we can construct a graph $G$ on vertex set $\{1,2, \ldots, n\}$ by joining the special vertices of the $G_{i}$ into a path, in the given order, by using the standard path construction:

$$
\eta_{1,2}\left(\rho_{1 \rightarrow 2}\left(\rho_{2 \rightarrow 3}\left(\ldots \eta_{1,2}\left(\left(\rho_{1 \rightarrow 2}\left(\rho_{2 \rightarrow 3}\left(\eta_{1,2}\left(\rho_{1 \rightarrow 2}\left(G_{1}\right) \oplus G_{2}\right)\right)\right) \oplus G_{3}\right) \ldots\right)\right) \oplus G_{\left\lfloor\frac{n}{k+1}\right\rfloor}\right)\right.
$$

and finally adding as isolated vertices any vertices in the smaller set.

Since a path has only two automorphisms, this process can construct the same labelled graph $G$ in just two ways: the other being of course to take the ordered partition and the sequence of graphs in the reversed orders.

It follows that the number of distinct graphs that can be constructed in this way is at least

$$
\left(\begin{array}{c}
n \\
k+1, \ldots, k+1
\end{array}\right) 2^{\left(\begin{array}{c}
k \\
2
\end{array}\right) \frac{n}{k+1}}>\frac{n !}{(k+1) ! \frac{n}{k+1}} 2^{\left(\begin{array}{c}
k \\
2
\end{array}\right)\left\lfloor\frac{n}{k+1}\right\rfloor-1}>n !\left(\frac{2^{\frac{k-2}{2}}}{k+1}\right)^{n},
$$

as required.

\subsection{Slow properties of unbounded clique-width}

\subsubsection{Unit interval graphs}

A graph $G$ is a unit interval graph if it is possible to choose for each vertex $x$ of $G$ an interval $I_{x}$ of unit length on the real line, such that $x y$ is an edge of $G$ if and only if the intervals $I_{x}$ and $I_{y}$ intersect.

Golumbic and Rotics [12] showed that this class has unbounded clique-width (and Lozin [14] showed that it is an inclusion-minimal hereditary class with unbounded cliquewidth). It is clear that if we have a unit interval representation of $G$, and $G^{\prime}$ is an induced subgraph of $G$, then taking the unit interval representation of $G$ and removing intervals corresponding to vertices in $V(G)-V\left(G^{\prime}\right)$ yields a unit interval representation of $G^{\prime}$, so the unit interval graphs form a hereditary class.

Theorem 4 The class of unit interval graphs has speed at most $n ! 4^{n}$. 
Proof. Let $G$ be any unit interval graph on vertex set $\{1,2, \ldots, n\}$, and fix a unit interval representation of $G$. Each interval has a start point and an end point (moving along the real line), so we can record a string of length $2 n$ consisting of $n$ symbols $\mathrm{S}$ and $n$ symbols E giving the order along the real line in which the starts and ends of intervals occur, and separately record the permutation $\sigma$, where the start point of the interval corresponding to vertex $i$ is the $\sigma(i)$-th start point encountered. Since the intervals are of unit length the same permutation gives the order in which the end points appear. Although we cannot reconstruct the unit interval representation from this recorded information, we can reconstruct the intersections of intervals and hence $G$.

It follows that there are at most as many unit interval graphs on $n$ vertices as there are choices of permutations of $n$ and $2 n$-element strings using two symbols: namely $n ! 2^{2 n}$.

\subsubsection{Bipartite permutation graphs}

The class of bipartite permutation graphs is another class of unbounded clique-width [7]. It is known that every bipartite permutation graph $G$ is biconvex, meaning that the vertices in each part of $G$ can be ordered so that the neighborhood of each vertex $v \in V(G)$ forms an interval in the opposite part, i.e., the neighbors of $v$ appear consecutively in the order.

Theorem 5 The class of bipartite permutation graphs has speed at most $n ! 28^{n}$.

Proof. Given a bipartite permutation graph $G$ with $n$ vertices and a biconvex representation of $G$, we record the graph as follows. First, we list the vertices of $G$ in the lower part in order, then place a separator, and then list the vertices of the upper part of $G$ in order. This gives us $n ! n$ possible records. This information however does not tell us anything about adjacencies of vertices in different parts. To record this information, for each vertex $v$ in the lower part we surround its neighborhood in the upper part with two parentheses indicating the beginning and the end of the interval. The record now consists of a list of vertices in the lower part, a separator, and a list of vertices in the upper part containing at most $n$ pairs of parentheses. It is not difficult to see that this record completely defines

the graph. An easy upper bound on the number of such strings is $n ! n 3^{3 n}$, which does not exceed $n ! 28^{n}$ for sufficiently large $n$.

\section{Open problems}

We leave two open questions.

First, it would be of some interest to determine more exactly the rate of growth of $T(k)_{n}$. This problem is likely to be solvable by generating function methods.

Second, we give one further function. Let

$$
f(n)=\min \left\{P_{n}: \mathcal{P} \text { is a hereditary property with unbounded clique-width }\right\} .
$$


The intent of this function is to specify exactly where the transition from structurally simple graph classes to complex ones occurs. We have shown that $f(n) \leq n ! 4^{n}$; but we do not know how close to the Bell number $B_{n}$ this function actually is. In fact, we do not even know that $f(n)>B_{n}$ is true, though we conjecture that it is. Our difficulties in proving this stem from essentially the same source as the difficulties Balogh, Bollobás and Weinreich [5] encounter in attempting to classify minimal hereditary graph classes with speeds exceeding the Bell number; we reiterate their call for more research in this area.

It would be interesting to know more about the function $f(n)$. There are several open issues: is there a minimal speed class of unbounded clique-width (whose speed is $f(n)$ for all sufficiently large $n$ ), and if so is it unique? Is $f(n)$ a function of the form $n ! \Theta(1)^{n}$ or is it true that $\frac{1}{n} \log \frac{n !}{f(n)} \rightarrow \infty$ as $n \rightarrow \infty$ ? Finally, is it possible to extend Balogh, Bollobás and Weinreich's classification to classes with speeds up to $f(n)$ ?

\section{Acknowledgements}

We are grateful to the anonymous referee for many suggestions that improved the presentation of the paper; and for drawing our attention to the importance of the function $f(n)$.

\section{References}

[1] V. Alekseev, On lower layers of the lattice of hereditary classes of graphs, Diskretn. Anal. Issled. Oper. Ser. 1 Vol. 4 (1997), no. 1, 3-12 (in Russian).

[2] P. Allen, Forbidden induced bipartite graphs, J. Graph Theory, to appear.

[3] J. Balogh, B. BollobÁs and D. Weinreich, The speed of hereditary properties of graphs, J. Combin. Theory Ser. B 79 (2000) 131-156.

[4] J. Balogh, B. Bollobás and D. Weinreich, The penultimate range of growth for graph properties, European J. Combin 22 (2001) 277-289.

[5] J. Balogh, B. Bollobás and D. Weinreich, A jump to the Bell number for hereditary graph properties, J. Combin. Theory Ser. B 95 (2005) 29-48.

[6] B. Bollobas, Extremal graph theory, London: Acad. Press, 1978.

[7] A. BrandstäDt and V.V. LozIn, On the linear structure and clique-width of bipartite permutation graphs, Ars Combinatoria, 67 (2003) 273-289.

[8] B. Courcelle, J. Engelfriet and G. Rozenberg, Handle-rewriting hypergraphs grammars, J. Comput. System Sci. 46 (1993) 218-270.

[9] B. Courcelle, J.A. Makowsky and U. Rotics, Linear time solvable optimization problems on graphs of bounded clique-width, Theory Comput. Systems, 33 (2000) $125-150$.

[10] B. Courcelle and S. Olariu, Upper bounds to the clique-width of a graph, Discrete Applied Math. 101 (2000) 77-114. 
[11] P. Erdős and J. Spencer, Probabilistic methods in combinatorics, Probability and Mathematical Statistics, Vol. 17. Academic Press, New York-London, 1974.

[12] M. Golumbic and U. Rotics, On the clique-width of some perfect graph classes, Int. J. Found. Comp. Sci. 11 (2000) 423-443.

[13] M. Kaminski, V. Lozin and M. Milanic, Recent developments on graphs of bounded clique-width, Discrete Applied Mathematics, accepted.

[14] V. Lozin, From tree-width to clique-width: excluding a unit interval graph, Lecture Notes in Computer Science 5369 (2008) 872-883.

[15] V. Lozin and D. Rautenbach, On the band-, tree- and clique-width of graphs with bounded vertex degree, SIAM J. Discrete Mathematics 18 (2004) 195-206.

[16] V. Lozin and J. Volz, The clique-width of bipartite graphs in monogenic classes, Int. J. Found. Comp. Sci. 19 (2008) 477-494.

[17] F. Lazebnik, V.A. Ustimenko and A.J. Woldar, A New Series of Dense Graphs of High Girth, Bulletin of the AMS, 32 (1995) 73-79.

[18] J.A. Makowsky and U. Rotics, On the clique-width of graphs with few $P_{4}$ 's, International J. Foundations of Computer Science, 10 (1999) 329-348.

[19] S. Norine, P. Seymour, R. Thomas and P. Wollan, Proper minor-closed families are small, J. Combinatorial Theory Ser. B, 96 (2006) 754-757.

[20] E.R. Scheinerman and J. Zito, On the size of hereditary classes of graphs, $J$. Combin. Theory, Ser. B 61 (1994) 16-39.

[21] J. P. Spinrad, Nonredundant 1's in $\Gamma$-free matrices, SIAM J. Discrete Math. 8 (1995) 251-257. 